\title{
Hepatitis B virus screening in patients with non-Hodgkin lymphoma in clinical practice in Poland - a report of the Polish Lymphoma Research Group
}

\author{
Ewa Kalinka1, Joanna Drozd-Sokołowska², Anna Waszczuk-Gajda², Joanna Barankiewicz³, \\ Elżbieta Zalewska ${ }^{4}$, Igor Symonowicz ${ }^{1}$, Ewa Lech-Marańda ${ }^{3}$
}

\author{
${ }^{1}$ Department of Oncology, Polish Mother's Memorial Hospital - Research Institute, \\ Lodz, Poland \\ ${ }^{2}$ Department of Hematology, Oncology and Internal Diseases, Warsaw Medical \\ University, Warsaw, Poland \\ ${ }^{3}$ Department of Hematology, Institute of Hematology and Transfusion Medicine, \\ Warsaw, Poland \\ ${ }^{4}$ Statistical Methods Unit, University of Lodz, Lodz, Poland
}

Submitted: 8 June 2019

Accepted: 24 June 2019

Arch Med Sci 2020; 16 (1): 157-161

DOI: https://doi.org/10.5114/aoms.2019.86761

Copyright @ 2019 Termedia \& Banach

\begin{abstract}
Introduction: The risk of HBV reactivation is important in lymphoma patients receiving immunosuppressive chemotherapy containing steroids or anti-CD2O antibodies. We aimed to establish the prevalence of HBV Ag and anti-HBc serologic positive results in patients with non-Hodgkin lymphoma $(\mathrm{NHL})$ and chronic lymphocytic leukemia (CLL) in Poland before anti-CD20 therapy initiation; to assess the frequency of insufficient HBV screening; and to assess the association between inadequate HBV screening and diagnosis according to the WHO classification and age or gender.

Material and methods: We retrospectively collected data from 805 patients with non-Hodgkin lymphoma and chronic lymphocytic leukemia treated in 2016-2018.

Results: We found a positive result of HBsAg in 13 (1.16\%), and a negative result in $633(78.64 \%)$ patients. The test was not done in $159(19.75 \%)$ patients. In the HBsAg negative subgroup of 633 patients, we found that the anti-HBc was positive in 52 (8.22\%), negative in $303(47.87 \%)$ and not done in 278 patients. In 136 out of 805 (16.9\%) patients diagnostics tests were not performed before therapy initiation. We found that age is significantly associated ( $p=0.0002)$ with the lack of HBV infection screening, and in CLL this risk is significantly $(p=0.024)$ higher (by $49 \%)$ compared with other WHO diagnosis subgroups.

Conclusions: In Polish lymphoma patients the incidence of positive HBsAg and/or anti-HBC results is consistent with the prevalence in the United States or Australia. The adherence to appropriate HBV screening guidelines in Polish centers is not sufficient. We should intensify educational strategies in the global oncohematologic medical community.
\end{abstract}

Key words: screening, chronic lymphocytic leukemia, hepatitis B virus, nonHodgkin lymphoma, anti-CD20 treatment.

\section{Introduction}

It is estimated that there are 350 million people chronically infected by hepatitis $\mathrm{B}$ virus (HBV) in the world [1]. Most of these carriers are

\author{
Corresponding author: \\ Ewa Kalinka MD, PhD \\ Department of Oncology \\ Polish Mother's \\ Memorial Hospital \\ - Research Institute \\ 281/289 Rzgowska St \\ 93-338 Lodz, Poland \\ Phone: +48 530770073 \\ E-mail: ewakalinka@wp.pl
}


inactive in terms of viral replication or symptoms, but the risk of HBV reactivation is important in lymphoma patients receiving immunosuppressive chemotherapy, especially containing steroids and/ or anti-CD-20 antibodies, that can reduce B cell numbers and antibody levels [2]. Huang et al. analyzed a group of lymphoma patients and resolved hepatitis B and they found that undetectable HBV viral load before chemotherapy did not confer reactivation-free status [3]. After rituximab-based therapy, the incidence of $\mathrm{HBV}$ reactivation in patients with lymphoma and resolved hepatitis $B$ is reported up to $23.8 \%$ [4]. Thus, appropriate screening and prophylaxis strategies need to be implemented in high-risk populations. The results of multiple studies have demonstrated that antiviral prophylaxis interventions can reduce the risk of HBV reactivation [3]. Hence, appropriate identification of the population at risk of HBV reactivation remains crucial in order to reduce the incidence of this complication in lymphoma patients.

In 2015, the American Gastroenterology Association (AGA) published guidelines on the prevention of hepatitis $B$ virus reactivation during immunosuppressive drug therapy. AGA defined the high-risk group as anticipated incidence of $\mathrm{HBV}$ reactivation in $>10 \%$ of cases. This group includes: 1) HBsAg-positive/anti-HBc-positive or HBsAg-negative/anti-HBc-positive patients treated with $B$ cell-depleting agents (for example rituximab, ofatumumab) and 2) HBsAg-positive/ anti-HBc-positive patients treated with anthracycline derivatives (for example, doxorubicin, epirubicin) and 3) HBsAg-positive/anti-HBc-positive patients treated with moderate-dose (10-20 mg prednisone daily or equivalent) or high-dose (> $20 \mathrm{mg}$ prednisone daily or equivalent) corticosteroids daily for at least 4 weeks. For these high-risk patients the AGA recommended antiviral prophylaxis that should be continued at least 6 months after discontinuation of immunosuppressive therapy and at least 12 months for B-cell depleting agents [5]. In 2014, Seto et al. conducted a study in lymphoma patients HBsAg-negative, anti-HBc-positive with undetectable serum HBV DNA $(<10 \mathrm{IU} / \mathrm{ml})$ treated with rituximab-based regimens, demonstrating that a high rate of $\mathrm{HBV}$ reactivation was observed in this population, with the risk of reactivation significantly higher in antiHBs-negative patients. Periodic HBV DNA monitoring was an effective strategy in preventing HBV-related complications [6].

The aim the study was to: (1) establish the prevalence of $\mathrm{HBs} \mathrm{Ag}$ and anti-HBc serologic positive results in patients with non-Hodgkin lymphoma and chronic lymphocytic leukemia in Poland before anti-CD20 therapy initiation, (2) to assess the frequency of insufficient HBV screening, (3) to assess the association between inadequate HBV screening and diagnosis according to the WHO classification and age or gender.

\section{Material and methods}

We retrospectively collected data in 4 hematology or oncology centers cooperating within the Polish Lymphoma Research Group from 805 patients diagnosed with non-Hodgkin lymphoma and chronic lymphocytic leukemia treated in 2016-2018, with a median age of 65. Most of the patients (over 90\%) were treated with rituximab containing regimens. Data were collected anonymously and consisted of demographic data such as age, gender, ethnicity, WHO diagnosis, and laboratory results of $\mathrm{HBsAg}$, anti-HBc, and anti-HBs blood test if available.

For the purpose of analysis we categorized four sub-groups: 1) diagnoses of aggressive and transformed lymphoma (including diffuse large B-cell lymphoma (DLBCL), Burkitt lymphoma, transformed indolent lymphomas into DLBCL), 2) chronic lymphocytic leukemia, 3) indolent lymphoma (including follicular lymphoma, plasmacytic lymphoma, marginal zone lymphoma and small lymphocytic lymphoma) and 4) other (including mantle cell lymphoma, nasal T/NK-cell lymphoma).

\section{Statistical analysis}

The nominal data were provided as absolute numbers and/or percent values. Making use of cross tabulations, the distribution of observations was presented in terms of several features simultaneously. For continuous variables the median and interquartile range (IQR) were calculated with the arithmetic mean and standard deviation (SD). The analysis of factors influencing non-performance of the complete examinations was assessed by means of univariate and multivariate logistic regression. The variables which turned out to be significant in univariate comparisons $(p<0.1)$ were included in the multivariate model. Results were considered statistically significant for $p<0.05$. Statistical analyses were performed by means of the Statistica 12.0 (StatSoft, Poland) program.

\section{Results}

Eight hundred and five Caucasian patients with a median age of 65 years $(56-73, I Q R=8.5), 100 \%$ of Caucasian ethnicity, 378 (47\%) female and 427 (53\%) male subjects were enrolled. Diagnoses according to the WHO classification were distributed as in Table I.

We found a positive result of HBsAg in 13 (1.16\%) patients and a negative result in 633 (78.64\%) patients. The test was not done in 159 (19.75\%) 
patients. Among patients with negative HBsAg the anti-HBc result was positive in 52 (8.22\%) patients, negative in 303 (47.87\%) patients and not done in 278 patients.

Logistic regression was further performed to study the relationship between the results of specific tests and chosen patients' characteristics. We were not able to identify an association between positive HBsAg result and any of the following factors: WHO diagnosis, gender or age (Table II). Similarly, in the group of HBsAg negative and anti$\mathrm{HBC}$ positive patients we did not find an association between the above-mentioned factors and the positive results. There was also no association between the lack of anti-HBC testing in HBsAg negative patients and the WHO diagnosis, gender or age (Table III).

Then, we found that in 136 out of 805 (16.9\%) patients no diagnostics tests were performed before therapy initiation. We then analyzed using logistic regression whether the lack of basic HBV infection screening result was associated with one of the above-mentioned factors. We found that age was significantly associated $(p=0.0002)$ with the lack of HBV infection screening, showing that each year of a patient's age increased the risk of lack of screening by $3 \%$. Additionally, we found that the risk of lack of HBV infection screening is significantly ( $p=0.024$ ) higher (by $49 \%$ ) in patients with CLL when compared to other WHO diagnosis subgroups. The results are presented in Table IV.

\section{Discussion}

Identification of patients with HBV infection is the most important step in preventing HBV reactivation as a result of anticancer therapies in lymphoma patients with special attention to those initiating immunosuppressive regimens with antiCD20 monoclonal antibodies.

In this study, we analyzed a cohort of lymphoma and CLL patients undergoing systemic therapy, including anti-CD20 monoclonal antibodies in $90 \%$ of cases, and assessed screening for HBV infection. The main findings were that the overall frequency of testing for screening purposes was low, with $16.9 \%$ of the patients not being tested at all for HBV before initiation of therapy and an ad-

Table I. Diagnosis according to WHO classification

\begin{tabular}{|c|c|}
\hline WHO diagnosis & $\begin{array}{c}\text { Number } \\
\text { of patients (\%) } \\
N=805\end{array}$ \\
\hline Diffuse large B-cell lymphoma & $265(32.9)$ \\
\hline Follicular lymphoma & $68(8.4)$ \\
\hline Nodal marginal-zone lymphoma & $33(4.1)$ \\
\hline MALT lymphoma & $15(1.9)$ \\
\hline Mantle cell lymphoma & $80(9.9)$ \\
\hline Small lymphocytic lymphoma & $34(4.2)$ \\
\hline $\begin{array}{l}\text { Waldenstrom macroglobulinemia/ } \\
\text { lymphoplasmacytic lymphoma }\end{array}$ & $19(2.4)$ \\
\hline Chronic lymphocytic leukemia & $199(24.7)$ \\
\hline Burkitt lymphoma & $6(0.8)$ \\
\hline DLBCL/HL & $6(0.8)$ \\
\hline DLBCL/BL & $12(1.6)$ \\
\hline $\mathrm{FL} / \mathrm{DLBCL}$ & $10(1.2)$ \\
\hline Other & $9(1.1)$ \\
\hline CLL/DLBCL & $4(0.5)$ \\
\hline Splenic marginal lymphoma & $21(2.6)$ \\
\hline $\begin{array}{l}\text { Primary mediastinal large B-cell } \\
\text { lymphoma }\end{array}$ & $22(2.7)$ \\
\hline MZL/DLBCL & $2(0.2)$ \\
\hline
\end{tabular}

$D L B C L$ - diffuse large B-cell lymphoma, MALT - mucosa associated lymphoid tissue, DLBCL/HL - B-cell lymphoma, unclassifiable, with features intermediate between DLBCL and classical Hodgkin lymphoma, DLBCL/BL - B-cell lymphoma, unclassifiable, with features intermediate between $D L B C L$ and Burkitt lymphoma, $F L /$ $D L B C L-D L B C L$ transformed from follicular lymphoma, CLL/DLBCL - DLBCL transformed from chronic lymphocytic leukemia, MZL/ $D L B C L-D L B C L$ transformed from marginal zone lymphoma.

Table II. Risk of positive HBsAg result depending on analyzed factors - results of univariate logistic regression

\begin{tabular}{|llccc|}
\hline Factor & & Odds ratio & 95\% confidence interval & P-value \\
\hline WHO & Aggressive and transformed lymphoma & 1.185 & $0.516-2.724$ & 0.689 \\
\cline { 2 - 6 } & Chronic lymphocytic leukemia & 0.635 & $0.2-2.017$ & 0.441 \\
\cline { 2 - 6 } & Indolent lymphoma & 0.993 & $0.362-2.721$ & 0.989 \\
\cline { 2 - 6 } & Other $(5,9,13)$ & 1.338 & $0.419-4.271$ & 0.623 \\
\hline Gender & Female & 0.838 & $0.477-1.471$ & 0.538 \\
\cline { 2 - 6 } & Male & 1.193 & $0.68-2.1$ & $0.951-1.024$ \\
\hline Age & & 0.986 & & 0.469 \\
\hline
\end{tabular}

Result statistically significant for $p<0.05$ 
Table III. Determination of positive anti-HBc result in patients with a negative HBsAg test depending on analyzed factors - results of univariate logistic regression

\begin{tabular}{|llccc|}
\hline Factor & & Odds ratio & 95\% confidence interval & $P$-value \\
\hline WHO & Aggressive and transformed lymphoma & 1.082 & $0.688-1.702$ & 0.731 \\
\cline { 2 - 5 } & Chronic lymphocytic leukemia & 0.894 & $0.52-1.536$ & 0.685 \\
\cline { 2 - 5 } & Indolent lymphoma & 1.272 & $0.774-2.01$ & 0.343 \\
\cline { 2 - 5 } & Other & 0.813 & $0.396-1.667$ & 0.571 \\
\hline Gender & Female & 0.989 & $0.74-1.307$ & 0.908 \\
\cline { 2 - 5 } & Male & 1.017 & $1.77-1.35$ & 0.069 \\
\hline Age & & 1.021 & $0.998-1.043$ & \\
\hline
\end{tabular}

Result statistically significant for $p<0.05$.

Table IV. Determination of chance to perform proper minimal HBV screening (HBsAg and anti-HBC) depending on analyzed factors - results of univariate logistic regression

\begin{tabular}{|llccc|}
\hline Factor & & Odds ratio & 95\% confidence interval & $P$-value \\
\hline WHO & Aggressive and transformed lymphoma & 0.760 & $0.557-1.036$ & 0.083 \\
\cline { 2 - 6 } & Chronic lymphocytic leukemia & 1.495 & $1.056-2.118$ & $0.024^{*}$ \\
\cline { 2 - 6 } & Indolent lymphoma & 0.912 & $0.636-1.308$ & 0.617 \\
\cline { 2 - 5 } & Other & 0.965 & $0.604-1.542$ & 0.881 \\
\hline Gender & Female & 0.96 & $0.790-1.167$ & 0.680 \\
\cline { 2 - 5 } & Male & 1.042 & $1.014-1.046$ & $0.0002^{*}$ \\
\hline Age & & 1.03 &
\end{tabular}

${ }^{\star}$ Result statistically significant for $p<0.05$.

ditional $34.5 \%$ of patients in whom screening was limited to a negative HBsAg result. Thus, 51.4\% of non-Hodgkin lymphoma and CLL patients were not screened properly in order to minimize the risk of HBV reactivation during systemic treatment.

There is an important difference between guideline recommendations by the liver societies and those by the oncology professionals with regard to HBV screening prior to chemotherapy, but recommendations for lymphoma patients, especially starting anti-CD20 therapy, are clear and indicate the need of at least serologic screening for this entire population. As shown by Wi et al., the proportion of hematologic patients treated in Mayo Clinic in Rochester screened for HBV increased from $32.7 \%$ in $2006-2008$ to $40.6 \%$ in $2009-2011$ ( $p<$ 0.01 ) [7]. Thus, our results show that only $48.6 \%$ of lymphoma and CLL patients treated during 2016-2018 in Poland were screened properly for $\mathrm{HBV}$ reactivation risk, which is in accordance with the results obtained in the Mayo Clinic. In our study gender was not a significant risk factor for insufficient HBV screening, while in the Mayo Clinic group male patients were more likely to have HBV screening. We found that age is significantly associated with the lack of HBV infection screening, which is consistent with the results obtained by Wi et al. [7]. In 2018, an Australian group conducted a study to determine: the adherence to the local protocol for HBV screening; the HBV infection prevalence in patients receiving rituximab; and outcomes of patients at risk of HBV reactivation. Adherence was assessed by documentation of both HBsAg and HBCAb prior to rituximab initiation. Two hundred and nine (47.7\%) failed to receive appropriate HBV screening, 86 (19.6\%) had neither HBsAg nor HBCAb performed, and 119 (27.2\%) had only HBsAg performed [8]. These results are consistent with our observations, showing that insufficient HBV screening in lymphoma patients is a global problem requiring probably generalized educational efforts in the oncohematologic community.

In our analysis, in the HBsAg negative subgroup of 633 patients, we found an anti-HBc positive result in $8.22 \%$ of these patients, anti-HBc negative in $47.87 \%$ of patients and not done in $43.91 \%$ of patients. As shown in a meta-analysis published in 2017 [9], including a total of 1312 HBsAg-negative/HBC Ab-positive lymphoma patients treated with rituximab-containing chemotherapy, the $\mathrm{HBV}$ reactivation rate is $9.0 \%$ in retrospective and prospective studies, which provides evidence that potentially the risk of $\mathrm{HBV}$ reactivation in 
HBsAg-negative/HBc Ab-positive patients with rituximab treatment for lymphoma is substantial. This study is a rationale to intensify educational activities in the medical community treating lymphoma patients in order to incorporate the anti-HBc testing as a routine diagnostic procedure in HBsAg-negative lymphoma patients in daily clinical practice.

A total of 1962 patients with hematological malignancies were enrolled in a study in Taiwan. Of the 286 HBV carriers, 97 (33.9\%) developed hepatitis $B$ reactivation during or after chemotherapy. Of the 1676 patients with initial negative HBsAg counts, 41 (2.4\%) experienced hepatitis B reactivation and reverse seroconversion of $\mathrm{HB}$ $\mathrm{sAg}$, and lost their protective hepatitis B surface antibody (anti-HBs). Moreover, positive anti-HBc ( $p=0.013, \mathrm{OR}=0.070,95 \% \mathrm{Cl}: 0.009-0.571)$ was one of the independent risk factors of positive seroconversion of $\mathrm{HBsAg}$ in patients with hematological malignancy. This study provides a further rationale to support routine anti-HBc testing in all HBsAg negative patients with lymphoma. This study revealed that the incidence of HBV reactivation among the patients with different subtypes of hematological malignancy is similar [9, 10]. Thus, insufficient HBV infection screening in CLL patients in our study is not supported by scientific evidence.

Different screening strategies in oncology have different detection levels, as in colorectal cancer different endoscopic methods give an improved adenoma detection rate with implementation of novel technical methods [11]. Thus, more sensitive simple serologic detection of HBV infection could potentially decrease HBV reactivation risk in lymphoma and CLL patients.

In conclusion, in Polish lymphoma patients the incidence of positive $\mathrm{HBsAg}$ and/or anti-HBC results is consistent with the prevalence in the United States or Australia. The adherence to appropriate HBV screening guidelines in Polish centers is not sufficient but is similar to leading centers treating lymphoma, which provides further evidence to intensify educational strategies in the global oncohematologic medical community. Our study showed that we tend to omit proper HBV screening in elderly patients and in those with CLL more than non-Hodgkin lymphoma subtypes.

\section{Conflict of interest}

The authors declare no conflict of interest.

\section{References}

1. Lavanchy D. Hepatitis B virus epidemiology, disease burden, treatment, and current and emerging prevention and control measures. J Viral Hepat 2004; 11: 97-107.
2. Hsu C, Hsiung CA, Su IJ, et al. A revisit of prophylactic lamivudine for chemotherapy-associated hepatitis B reactivation in non-Hodgkin's lymphoma: a randomized trial. Hepatology 2008; 47: 844-53.

3. Huang YH, Hsiao LT, Hong YC, et al. Randomized controlled trial of entecavir prophylaxis for rituximab-associated hepatitis $B$ virus reactivation in patients with lymphoma and resolved hepatitis B. J Clin Oncol 2013; 31: 2765-72.

4. Yeo W, Chan TC, Leung NW, et al. Hepatitis B virus reactivation in lymphoma patients with prior resolved hepatitis B undergoing anticancer therapy with or without rituximab. J Clin Oncol 2009; 27: 605-11.

5. Reddy KR, Beavers KL, Hammond SP, Lim JK, FalckYtter YT. American Gastroenterological Association Institute guideline on the prevention and treatment of hepatitis b virus reactivation during immunosuppressive drug therapy. Gastroenterology 2015; 148: 215-9.

6. Seto WK, Chan TS, Hwang YY, et al. Hepatitis B reactivation in patients with previous hepatitis $B$ virus exposure undergoing rituximab-containing chemotherapy for lymphoma: a prospective study. J Clin Oncol 2014; 32: 3736-43.

7. Wi Cl, Loo NM, Larson JJ, et al. Low level of HBV screening among patients receiving chemotherapy. Clin Gastroenterol Hepatol 2015; 13: 970-5.

8. Hall SAL, Shaikh A, Teh K, et al. Hepatitis B screening before rituximab therapy: a multicentre South Australian study of adherence. Intern Med J 2018; 48: 936-43.

9. Tang Z, Li X, Wu S, et al. Risk of hepatitis B reactivation in HBsAg-negative/HBCAb-positive patients with undetectable serum HBV DNA after treatment with rituximab for lymphoma: a meta-analysis. Hepatol Int 2017; 11: 429-33.

10. Chen CY, Tien FM, Cheng A, et al. Hepatitis B reactivation among 1962 patients with hematological malignancy in Taiwan. BMC Gastroenterol 2018; 18: 6.

11. Matyja M, Pasternak A, Szura M, Wysocki M, Pędziwiatr $M$, Rembiasz K. How to improve the adenoma detection rate in colorectal cancer screening? Clinical factors and technological advancements Arch Med Sci 2019; 15: 424-33. 